\title{
Introduction : Gouvernance et grande vitesse
}

Robert Belot

\section{OpenEdition}

Journals

Electronic version

URL: http://journals.openedition.org/rge/5495

DOI: $10.4000 /$ rge.5495

ISSN: $2108-6478$

\section{Publisher}

Association des géographes de l'Est

Printed version

Date of publication: 1 October 2015

ISSN: 0035-3213

\section{Electronic reference}

Robert Belot, «Introduction : Gouvernance et grande vitesse », Revue Géographique de l'Est [Online], vol. $55 / n^{\circ} 3-4$ | 2015, Online since 29 October 2015, connection on 24 September 2020. URL : http:// journals.openedition.org/rge/5495; DOI : https://doi.org/10.4000/rge.5495

This text was automatically generated on 24 September 2020

Tous droits réservés 


\title{
Introduction : Gouvernance et grande vitesse
}

\author{
Robert Belot
}

1 On peut dire que la grande vitesse ferroviaire a été une « révolution » dans la mesure où elle a permis d'appréhender le ferroviaire autrement que comme un simple moyen de transport et d'accroissement de la mobilité. Elle a été regardée et appropriée par les politiques comme une aubaine de transformation et de dynamisation des territoires, comme une promesse de dépassement des frontières et d'accélération du processus d'européanisation, comme une assurance d'égalité sociale et territoriale mais aussi de rentabilité. Rarement un grand équipement n'aura été perçu, rêvé et vanté comme une incarnation de la modernité et comme l'incantation du progrès. Plus encore, une vitrine de la France à l'étranger. La grande vitesse ferroviaire fut autant une prouesse technique qu'un mythe collectif qui ne souffrait guère la contradiction. La France communiait dans le tout-TGV partout et pour tous. Or, depuis une quinzaine d'années, ce qui fut une grande ambition nationale est rattrapé par le principe de réalité. Nous sommes entrés dans l'ère du soupçon, marquée par l'évaluation de la distorsion qui s'est installée entre ce qui avait été dit et promis des effets escomptés et ce qui a effectivement eu lieu. De la Cour des Comptes aux universitaires, l'heure de la mise en examen critique semble avoir sonné. "Le rêve du tout-TGV a vécu : trop cher ", prédisait déjà l'hebdomadaire L'Express en mai 1996 suite au rapport Rouvillois. Le trentième anniversaire de la naissance $d u$ TGV a été marqué par des brûlots journalistiques. En juin 2013, alors que le TGV a atteint son deuxième milliard de voyageurs, un quotidien régional reprenait l'antienne : « Le Tout TGV réduit à peau de chagrin ».

2 Bien que le Grenelle de l'environnement prévoyait quelque 240 milliards de chantiers d'infrastructures de transport à l'horizon 2030, dont une grande part dévolue à la construction de lignes ferroviaires à grande vitesse, le Premier ministre Jean-Marc Ayrault adoptait alors les conclusions de la commission parlementaire « Mobilité 21. Pour un schéma national de mobilité durable ». Cette commission annonçait que « le modèle de développement ferroviaire est à revisiter ", et elle préconisait un report au- 
delà de 2030 des grands chantiers envisagés pour les LGV. La réalité budgétaire de la France, avec le déficit abyssal de 39 milliards d'euros du système ferroviaire français, ont eu raison d'un certain imaginaire trentenaire. Les politiques aujourd'hui font preuve de modestie et de réalisme : ils s'attachent à la modernisation des transports de proximité et « du quotidien ». Selon le journal Le Monde, l'accident de Brétigny, qui a fait sept morts le 12 juillet 2013, est regardé comme « la sanction d'un choix politique » vieux de plus de trente ans, «celui du tout-TGV, qui a fait la fierté de la France et porté les couleurs du pays dans le monde entier ». Le 1er septembre 2015, sur l'antenne de $\mathrm{BFM}$, le candidat socialiste à la Région île-de-France assurait que le nombre de passagers utilisant chaque jour par les RER A et $\mathrm{B}$ dans la région parisienne équivalait à celui de population transportée « dans toute la France par le TGV », son souci principal étant de moderniser les caténaires de la ligne $C$ parfois vieux de 80 ans...

La dernière mise en service d'une ligne à grande vitesse à ce jour est le premier tronçon de la LGV Rhin-Rhône : première phase de la branche Est, qui correspond au tronçon central (140 km sur $190 \mathrm{~km}$, pour la totalité de la branche Est) entre Dijon et Mulhouse. Le projet de recherche qui a abouti au colloque international des 10 et 11 juillet 2014 accueilli à l'ATRIA-Novotel de Belfort (" Grande vitesse ferroviaire \& gouvernance territoriale »), et donné lieu à ce numéro spécial de la Revue Géographique de l'Est, avait initialement pour objet l'étude de cette dernière LGV. Le laboratoire RECITS (EA $\mathrm{n}^{\circ} 7274$ ) de l'université de Technologie de Belfort-Montbéliard a été lauréat d'un appel d'offre de la région Franche-Comté qui lui a permis de mener à bien ce programme de recherche consacré à la gouvernance des projets ferroviaires de grande vitesse. Il répondait à une double ambition : d'abord, construire un cadre de compréhension de la genèse d'un projet de ligne à grande vitesse, identifier le rôle des différents acteurs impliqués et les modes d'appropriation du projet par les différents acteurs ; ensuite, questionner les effets du projet de LGV sur la gouvernance des territoires traversés et sur l'émergence annoncée d'une « métropole Rhin-Rhône ».

Le soutien de la Région Franche-Comté n'a pas seulement permis l'organisation matérielle du colloque : il est à l'origine du recrutement d'un post-doc qui a été chargé de procéder au dépouillement exhaustif et à la numérisation des archives de l'Association Trans-Europe-TGV-Rhin-Rhône-Méditerranée déposées aux archives départementales du Territoire de Belfort. Il y aura bientôt 30 ans que cette association de préfiguration a été créée ; elle aura été le bras armé de Jean-Pierre Chevènement, ancien ministre et ancien député-maire de Belfort, qui fut l'acteur-réseau de l'aventure de la LGV Rhin-Rhône. Nous avons pu avoir accès à son fonds personnel, conservé aux archives municipales de Belfort. Cette exploration de sources archivistiques, tout à fait conséquente, a permis l'extraction et le traitement de plus de plus de 3000 documents. Ces fonds ont été complétés par les archives Alst(h)om-Belfort déposées également aux archives départementale du Territoire de Belfort. D'autres sources archivistiques ont été explorées et une campagne d'entretiens a été réalisée (acteurs politiques nationaux ; politiques locaux ; industriels ; associations ; syndicats). La mise en œuvre de ce programme sur la LGV Rhin-Rhône a été initialisée à l'occasion du XLIXe colloque de l'Association de science régionale de langue française (Belfort, 9-11 juillet 2012) : « Industrie, villes et régions dans une économie mondialisée ». Membre du comité scientifique, RECITS a organisé une session et présenté un ensemble de communications. Une de ces communications (publiée dans la revue Recherche, Transports, Sécurité 2013) a lancé le vaste projet d'historisation de la LGV Rhin-Rhône structuré autour de trois axes : sa génétique, son portage politique et son impact 
territorial. Il s'agissait, dans un premier temps, et sur la base d'archives inédites, de révéler (car cette dimension a peu été revendiquée, sinon déniée) que la question du TGV Rhin-Rhône s'inscrit dans une relation partenariale séculaire avec la Suisse, plus particulièrement avec sa partie jurassienne et francophone.

5 En effet, dès le milieu du XIXe siècle, le paradigme helvétique s'insère au cœur des politiques de développement du ferroviaire dans cette marche de l'Est que constitue le Nord Franche-Comté. Une communauté de destin et d'intérêt entre des espaces de marge se consolide au gré des dossiers d'infrastructures majeures. L'épaulement franco-suisse a joué un rôle majeur dans le processus d'appropriation politique de la LGV. S'il répond à une volonté de mise en résonance de ces territoires à la culture transfrontalière ancienne, il correspond à la volonté plus récente d'inscription dans la dynamique des grandes transversales européennes (Nord-sud et Est-Ouest). Cet emboîtement des intérêts régionaux et transnationaux constitue la trame de ce projet, même si, initialement, semble dominer une tendance obsidionale autour de la lutte contre « l'enclavement » et « l'encerclement » et le tropisme de la radiale Suisse-Paris. Jean-Pierre Chevènement, l'inspirateur et l'acteur multi-scalaire de la LGV, a construit son discours primitif sur ce sentiment largement partagé. C'est pourquoi, dès l'origine, il a associé la Suisse et encouragé son désir d'appariement au réseau français de la grande vitesse. Son discours s'est ensuite européanisé. En 1993, il explique : " C'est un maillon clé du réseau européen des trains à grande vitesse. Aussi bien dans le sens Nord-Sud (Francfort-Strasbourg-Lyon-Méditerranée) qu'Est-Ouest (Suisse-Paris). » L'avenir dira si cette ambition était un mythe, car le projet de branche Sud, vers Lyon, qui visait à renforcer les échanges entre le nord et le sud de l'Europe, entre le HautRhin et l'arc méditerranéen, semble bien compromis aujourd'hui.

6 La troisième phase de ce programme de recherche, à venir, vise à interroger les effets réels de cette nouvelle ligne tant sur les territoires (effets économiques notamment) que sur le mode de gouvernance inter-collectivités (effets politiques), en allant jusqu'aux pratiques mobilitaires. Il s'agit finalement de tenter de mesurer l'écart entre les attentes et les croyances du discours politique et la réalité des faits, sachant que l'horizon méta-heuristique de cette recherche serait de cerner l'enjeu que représente un grand équipement de ce type dont l'originalité (et la difficulté) est de ne pas être porté par une grande métropole régionale. C'est pourquoi l'Association Trans-EuropeTGV-Rhin-Rhône-Méditerranée, par compensation, se devait d'avoir un spectre partenarial très large et surinvestir dans la rhétorique européenne. Dans les années 1990, Chevènement répète à l'envi : " L'association que je préside rassemble cinq Régions françaises, deux Länder allemands, de nombreux cantons suisses alémaniques et romans et la Catalogne. » C'est pourquoi aussi le discours politique a largement puisé dans le thème prophétique du TGV comme événement préparant l'avènement d'un territoire à l'identité propre en lieu et place d'un simple couloir, peu soucieux de faire le départ le départ entre le mythe et la réalité. Les géographes, dans le sillage ouvert par Raymond Woessner, se sont depuis attaqués à la question, montant combien il est difficile de transformer un "axe » en " réseau » s'agissant d'un espace qui, entre l'Alsace et la Bourgogne, n'a pas d'unité régionale ...

7 Le colloque avait justement pour ambition de resituer cette problématique régionale dans un cadre plus vaste, dépassant l'espace français et misant sur l'intérêt des approches comparatistes. Les articles sélectionnés ici donnent un riche aperçu de l'intérêt de cette approche qui permettra de relancer notre enquête. C'est l'essence 
même de la recherche que de s'inscrire dans une démarche collective et de se nourrir des regards croisés. Les papiers sélectionnés de ce numéro, issus du colloque, témoignent pleinement de l'importance que le monde académique accorde aux enjeux multiples du phénomène de la grande vitesse ferroviaire.

8 L'objectif était de questionner les effets de la grande vitesse sur la gouvernance des territoires traversés en comparant les expériences (en France et à l'étranger) mais aussi en confrontant l'attente des décideurs publics et les résultats. Car l'importance (supposée) des lignes ferroviaires à grande vitesse sur le développement territorial et le coût qu'elles représentent ont fait que les acteurs locaux se sont largement investis dans ce dossier. Qu'il s'agisse des collectivités territoriales qui se battent pour obtenir une gare ou au contraire éviter le passage des rails sur leur territoire, des milieux économiques qui voient dans la grande vitesse le support d'un développement futur, ou inversement des opposants qui remettent en cause son impact sur l'environnement ou sur les mobilités, les acteurs locaux s'impliquent fortement dans ces projets, au point que Nathalie Kosciusko-Moriset, ministre de l'Écologie lors des Assises du ferroviaire (2011), a pu dire que " le désir de TGV est peut-être davantage celui des élus que celui de nos concitoyens ". Les travaux académiques ont déjà largement interrogé les évolutions territoriales liées à la grande vitesse, qu'elles soient économiques (sur le prix de l'immobilier, l'emploi, l'attractivité touristique...) ou spatiales (sur la recomposition des mobilités, la métropolisation...), en particulier en interrogeant la croyance dans les « effets structurants » des infrastructures de transport et les mythes qu'elle suscite. Ils ont montré la complexité du lien entre grande vitesse et développement économique local. Pour autant, il reste un champ assez inexploré jusque-là : celui des transformations des modes de gouvernance. Quel est l'impact de la grande vitesse ferroviaire sur la manière dont les collectivités se comportent entre elles ? La grande vitesse peut-elle être un outil de la recomposition d'une nouvelle gouvernance entre les territoires traversés ? Autant de questions qui sont abordées dans ce numéro à travers des analyses relevant de plusieurs de disciplines (géographie, histoire, économie, sciences politiques).

9 De ces analyses, une tonalité commune apparaît qui tend à souligner le décalage existant entre le discours sur une nouvelle gouvernance locale espérée ou rêvée, et la réalité du terrain marquée par la résistance de concurrences fortes ou de rivalités anciennes et le constat d'un impact mitigé de l'effet TGV sur les territoires et leur gestion.

S'agissant de la LGV Rhin-Rhône, Raymond Woessner n'hésite à faire dans l'iconoclastie en posant la question : " Le TGV Rhin-Rhône était-il nécessaire ? " Question qui aurait paru sacrilège il y a seulement quelques années! Si l'Alsace gagne en connectivité, en termes de temps de parcours, l'apport n'est pas encore décisif. Pour le meilleur temps, c'est le nord-Franche-Comté qui arrive vainqueur : Belfort et Montbéliard sont les seules grandes bénéficiaires sur ce plan. Mais la question se pose de savoir si la création des gares d'Auxon (Besançon) et de Mérou (BelfortMontbéliard), en zones rurales, aura un effet d'aubaine et sera de nature à constituer un pôle périurbain de redynamisation ? C'était le vœu des élus. Mais rien n'est moins sûr. Il y a un grand risque « d'urbaniser mais sans urbanité ». L'association de portage a-t-elle atteint son objectif ? Certainement pas dans la mesure où la branche Sud « n'a ni financement, ni tracé, ni calendrier ". Mais c'est surtout l'ambition quasi eschatologique de faire naître une « métropole Rhin-Rhône » qui doit être révisée à la 
baisse. Les villes de la LGV entretiennent toujours aussi peu de relations, comme si l'espace n'était pas partagé, comme si le TGV avait été impuissant à changer les habitudes des navetteurs de l'axe Rhin-Rhône. La "systémogénèse » est à venir, tant résiste le temps long de l'univers centralisé à la française.

11 Seconde étude sur la LGV Rhin-Rhône, celle de Robert Belot, qui montre, sur la base des archives de l'Association Trans-Europe-TGV-Rhin-Rhône-Méditerranée pour la première fois explorée systématiquement, que cette réalisation n'aurait certainement pas pu voir le jour sans un activisme politique (local national et international) qui l'a finalement imposée à l'État. Cet équipement n'a pas été seulement soutenu par le discours politique ; il a été créé par lui et a permis de convaincre l'ensemble des acteurs (politiques, sociaux, industriels) de sa nécessité eu égard à " l'intérêt national » et européen qu'il était censé incarner, la population restant peu impliqué. Le cas de la LGV Rhin-Rhône illustre ce que l'on observe d'une manière plus générale : les grands projets d'équipements collectifs existent d'abord à travers le discours qu'on tient sur eux, un discours qui se présente comme une "fiction " argumentative, s'inscrivant dans une communauté de croyances, d'espérances, de mythes. Cette fiction a un caractère instituant et agissant car elle est un vecteur de légitimation du projet, un facteur d'adhésion au projet. Le discours politique sur la LGV relève donc en partie de " l'idéologie ». Il joue sur des régimes rhétoriques composites qui se compénètrent : technique / utopique ; rationnel / symbolique ; stratégique / opportuniste. Il manie des échelles multiples : du local (la crainte de la désindustrialisation du nord-FrancheComté) à l'Europe en passant par l'axe franco-suisse. Il charrie les doxas habituelles sur les effets supposés du TGV (désenclavement, rentabilité, attractivité, métropolisation de l'espace Rhin-Rhône...). C'est pourquoi Jean-Pierre Chevènement, le grand organisateur de ce "lobbying d'intérêt général ", a placé la communication au centre de sa " stratégie d'alliance " et d'influence. Ce discours a réussi à fédérer des collectivités (dont les intérêts n'étaient pas forcément convergents) et à accomplir cette prouesse de faire passer l'intérêt de la partie septentrionale de la région FrancheComté comme constituant un enjeu majeur pour l'ensemble des régions concernées, la Nation et l'Europe.

12 Une nouvelle gouvernance intra et inter-régionale a-t-elle pu émerger, comme le discours politique le laissait entendre ? Il est trop tôt pour l'analyser, d'autant que la réforme régionale en cours va modifier la donne en profondeur. De même s'agissant de l'enjeu géopolitique du départ à travers le lien franco-suisse. Les attentes du TGV comme instrument géopolitique concernent également la relation franco-espagnole. Les papiers de ce numéro consacré au cas espagnol ne doivent pas surprendre : depuis 2010, l'Espagne est le pays pourvu du plus grand kilométrage (2 $516 \mathrm{~km}$, contre 2036 pour la France) de lignes à grande vitesse d'Europe. Dans ce pays, le désir de TGV a partie liée au désir d'Europe.

13 La question doit enfin être posée de savoir si c'est l'Europe qui fait la grande vitesse ou si c'est la grande vitesse qui fait l'Europe? Tel est le sens de l'article d'Ander Audikana qui prolonge sa thèse de sociologie sur « la politisation de la grande vitesse espagnole » en s'attaquant à la construction d'un mythe politique et en montrant que la grande vitesse pourrait être analysée comme " une unité de mesure de la politique car, en partie, faire de la politique en Espagne a signifié pendant un certain temps construire des lignes à GV ». Son travail sur l'énonciation politique de la grande vitesse le conduit à considérer que celle-ci a pu, paradoxalement, "soulever et, la plupart de temps, 
renforcer les différences ou les identités territoriales ». Certes, la grande vitesse participe d'une dynamique d'européanisation de l'Espagne en termes de convergence de pratiques. Le symbole le plus clair est bien sûr l'adoption de l'écartement européen en ce qui concerne le transport international : elle a permis un accroissement de l'utilisation du chemin de fer, l'élaboration d'une politique en faveur de l'intermodalité, l'intégration des enjeux environnementaux, et même l'émergence d'une industrie ferroviaire espagnole. Pourtant, l'auteur fait valoir que le processus d'européanisation n'est pas exclusif d'une logique de "différenciation par rapport aux pratiques européennes ", et va jusqu'à évoquer une " dynamique d'hispanisation ». Tout se passerait comme si le développement de la grande vitesse avait été regardé comme le biais permettant d'accéder à des ressources matérielles (l'Espagne est le principal bénéficiaire des fonds européens en termes absolus, soit 45 milliards destinés à la grande vitesse) et symboliques. Bref, la question méritait d'être posée mais attend encore sa réponse : si le cadre européen a directement contribué au développement de la grande vitesse en Espagne et à la modernisation du ferroviaire, il est encore à démontrer que la grande vitesse (et ses possibilités de connexion avec les métropoles européennes) a été pour l'Espagne un " élément fondamental » de convergence avec l'Europe.

Des éléments de réponse peuvent être trouvés dans l'article d'éloïse Libourel. Elle a décidé d'appréhender la question par le biais du corridor ferroviaire méditerranéen, l'un des neuf « corridors multimodaux » formant le " réseau central » européen défini par la Commission européenne en 2013 des RTE-T (Réseaux Transeuropéens de Transport). Son étude est aussi une manière d'interroger les pratiques et les enjeux politiques, mais aussi de jeter l'attention sur le problème de la cohérence entre l'échelle locale, où se jouent peut-être les vrais enjeux, et l'échelle nationale et européenne, entre le fret ferroviaire (qui est au cœur du projet) et la grande vitesse (qui n'est pas centrale). Impliquant des acteurs-réseaux politiques très différents et des espaces transnationaux et régionaux multiples, ce projet est d'abord une autre manière de se représenter l'espace politique pour les Espagnols : le scénario d'une double ligne de passagers et de marchandises, de la frontière française à Algesiras (avec l'insertion de Valence, troisième ville espagnole), représente une nouveauté dans le réseau espagnol, très fortement polarisé autour de Madrid. Il s'agit à la fois de sortir de la logique radiale en imaginant un maillage progressif du territoire passant par les principales villes de province et reliant les grands ports espagnols, et de nouer une liaison vers l'Europe, dans une logique de "corridor ». Mais c'est un niveau de complexité nonpareil qui semble caractériser ce dossier qui témoigne de la multiplication des acteurs (institutionnels et non-institutionnels), des lieux de concertation et d'informations, et des échelons décisionnaires du fait de l'implication financière de l'Union européenne. Tout ce qui ne peut qu'affecter la gouvernance territoriale traditionnelle. Autre facteur de complexification, la revendication identitaire régionale face à l'autorité centrale, souvent contestée, et les tentatives d'appropriation de la grande vitesse à des fins de revitalisation urbaine où les lobbies publics et privés qui obligent le gouvernement central espagnol à penser autrement son action. Ainsi donc, derrière le consensus apparent se déploient des enjeux de gouvernance que cristallise le projet de corridor qui mêle dimension interrégionale et dimension transnationale, faisant intervenir des échelons de compétences très différents, dont les champs de souveraineté se recomposent au gré des rapports de force. 
15 La problématique de la transfrontiérité franco-espagnole et du TGV comme enjeu géopolitique reçoit un éclairage complémentaire grâce à Sophie Masson et Adenane Djigo. Le tronçon transfrontalier de la LGV Sud Europe-Méditerranée, entre Perpignan et Barcelone, s'inscrit dans une logique d'intégration européenne s'appuyant sur la mise en place d'un réseau ferroviaire européen à grande vitesse rapprochant les métropoles européennes. Mais elle répond aussi à une volonté politique de coopération inter-gouvernementale franco-espagnole, à travers la suppression de la discontinuité ferroviaire frontalière. L'enjeu ne relève pas que du champ symbolique : il est approprié par les acteurs des villes desservies, en particulier Perpignan, qui souhaitent développer une coopération transfrontalière de proximité. Mais les auteurs montrent que si les temps de parcours entre les gares de Perpignan, Figueras et Gérone se sont améliorés, l'accompagnement sous forme de mise en œuvre d'innovation de services tant en termes de dessertes de transport complémentaires (notamment en services de transports collectifs routiers transfrontaliers) ou encore d'offres touristiques coordonnées fait défaut. Cet exemple illustre une nouvelle fois les limites de la pensée magique du TGV et de ses supposés " effets structurants " lorsque les acteurs territoriaux ne se donnent pas pleinement « les moyens de transformer une intention en véritable plan d'actions de la coopération transfrontalière ».

Les recherches relatives à l'impact réel du TGV sur les territoires font l'objet de l'article de Sylvie Bazin-Benoît et Marie Delaplace. Les résultats des enquêtes pionnières qu'elles conduisent sur le cas de Reims ont fait avancer considérablement la compréhension générale du phénomène TGV et de ses « retombées » territoriales. Ici, elles mettent la focale sur la dimension touristique, qui est au cœur du discours politique relatif au grand équipement comme outil de développement et comme source de coopérations innovantes. En quoi la mise en service d'une desserte TGV peut-elle avoir un effet en termes d'attractivité ? Plus précisément, il s'agit de savoir, à partir d'exemples de villes de taille différente, si les nouvelles formes de gouvernance provoquées par l'avènement des dessertes ont réussi à avoir un impact sur le développement touristique. À l'évidence, on observe un effet positif de cette volonté de valorisation en termes d'accessibilité, à travers, notamment, l'amélioration de l'offre intermodale en sortie de gare, la mise en place d'une offre de paniers de biens mieux coordonnée sous formes de "packages » avec guichet et tarification uniques, des services hôteliers et de restauration adaptés, la création d'événementiels (comme à Reims ou au Mans). Mais si les villes de grande taille, bien positionnées par rapport à Paris et au réseau à grande vitesse européen, et bénéficiant d'un patrimoine connu, arrivent à tirer profit du TGV sur le plan touristique (Lille, par exemple), les villes moyennes et petites éprouvent des difficultés à capitaliser sur l'arrivée du TGV, en dehors de l'effet indirect d'image. On a même vu la fréquentation touristique de Charleville-Mézières connaître une baisse. C'est dire que les politiques de communication et une bonne desserte " peuvent accompagner l'attractivité de ces villes mais ne la créent pas ", d'autant que le tourisme (et notamment le tourisme d'affaires) est très sensible à la conjoncture. L'analyse des expériences de ces villes en matière de tourisme lié au TGV montre en particulier qu'il valorise surtout des lieux attirant certaines catégories de touristes, à revenus plutôt élevés, ayant des attentes fortes en matière d'offre touristique spécifique déjà connue et centrale. En fait, les rabattements des dessertes TGV vers les villes petites et moyennes profitent rarement au tourisme, le tourisme de court séjour étant peu adapté aux multi-destinations, sauf pour les grandes villes proches de Paris. 
17 La question des gares TGV et de leur environnement en lien avec la problématique de l'évaluation des dynamiques socio-économiques et spatiales commence à interpeler la recherche espagnole. Les premiers résultats correspondent à ce qui avait été pressenti dès les années 1990. C'est le cas de l'article de Carmen Vazquez Varela et José Maria Martinez Navarro qui s'attache à l'étude des transformations urbaines générées par la réalisation matérielle de l'infrastructure ferroviaire à grande vitesse (son degré d'intégration et de cohérence avec l'aménagement du territoire physique existant), ainsi que les projets urbains liés à celui-ci. L'analyse porte spécifiquement sur des sites à l'écart (entre 5 et $6 \mathrm{~km}$ ) des centres urbains de deux villes espagnoles moyennes : la gare de Cuenca (Fernando Zóbel), inaugurée en 2010 en périphérie de la ville et qui relie la ville de Madrid à Valence et Alicante, et une station située dans une municipalité de la province d'Alicante, sur la ligne TGV Madrid-Levante, à une distance de $6 \mathrm{~km}$ de la ville de Villena et 2,5 kilomètres de l'autoroute A-31, son inauguration ayant eu lieu le 17 juin 2013. Peut-être est-il tôt pour tirer des conclusions. Mais des signes et des tendances peuvent déjà conduire à des constats intéressants qui confirment ce qui est observé ailleurs. Ainsi, il est établi que la capacité du TGV à susciter une dynamique locale ou régionale " dépend du degré d'intégration territoriale de l'infrastructure ", de l'articulation avec les autres modes de transport, mais aussi et surtout d'une politique volontariste de transformation urbaine. Dans le cas de Cuenca et de Villena, condamnées à être des " gares perdues ", l'éloignement de la nouvelle infrastructure et le choix du site ont eu des effets contreproductifs : cette implantation n'a pas réussi à être intégrée dans un projet d'aménagement de la ville et du terrain, faute de consensus politique (confrontation entre la municipalité et le gouvernement régional, dissension entre l'opérateur de transport et les politiques) mais aussi du fait du séisme immobilier et de la crise financière et économique commencée en 2008.

Cette crise a bloqué d'autres projets. Comme celui d'implantation de la grande vitesse à Saragosse. Carmen Bellet Sanfeliu explique qu'il a été pensé bien en amont comme un instrument stratégique de rénovation urbanistique. Une association représentative des forces vives de la ville (EBROPOLIS) a été créée pour provoquer une véritable intelligence collective et anticipatrice. Après plusieurs scénarii d'implantations, il a été décidé de fermer la gare traditionnelle (pour faire naître une nouvelle aire urbaine centrale) et de créer une gare intermodale à l'ouest de la ville. Autour de cette gare avaient été prévus la construction d'immeubles d'affaires et de logements individuels, mais aussi une pépinière d'entreprises et un centre d'art et de technologie, vitrines de la modernité. Ce projet global peut-être trop ambitieux est victime des effets de la crise de 2008. Les acteurs doivent réécrire le scénario, avec des ambitions plus modestes.

La question des gares peut parfois prendre l'allure d'affrontements et provoquer une forte implication citoyenne. C'est le cas de Stuttgart, analysé par Anaïs Volin. À la faveur du projet européen de ligne ferroviaire Paris-Bratislava, les élus ont souhaité rénover la gare de cette ville-capitale du Land allemand du Bade-Wurtemberg. Les acteurs publics et privés ont mis en avant l'intérêt européen (liaison Est-Ouest) et la nécessité d'encourager des transports durables. Les élus locaux y voient une heureuse opportunité pour développer une politique urbaine rénovée. L'enterrement prévu des anciennes voies de chemin de fer ouvre des perspectives de création de logements en centre-ville et d'aménagement des nouveaux espaces verts alors que le quartier Europa, directement connecté à la gare centrale, deviendra un centre d'affaires. Ce projet d'ampleur ne peut pas se réaliser sans provoquer des perturbations profondes dans les 
habitudes des citoyens. Dès novembre 2009, la population manifeste son opposition, parfois de manière violente, ce qui provoque des réactions médiatiques locales et nationales très fortes. Le front du refus, qui prend des formes très organisées, critique l'insuffisance des consultations préalables, l'ampleur de l'effort financier et la gêne occasionnée ; il remet en cause l'intérêt de projet alors que des statistiques prouvent que les habitants du Land utilisent d'abord leur automobile pour leurs déplacements. Cette mobilisation de la société civile perturbe fortement le bon déroulement du chantier soutenu par les acteurs politiques nationaux. Ce genre d'opposition n'est pas unique, et a concerné la France dans les années 1990 (la ligne à grande vitesse LyonMarseille, par exemple). Mais elle a pris dans ce cas une ampleur singulière, dont témoigne le référendum de novembre 2008. Sa résonance dépasse les frontières allemandes : le collectif œuvrant contre les "Grands Projets Inutiles et Imposés » (GPII), créé en 2010 pour dénoncer les grands projets d'infrastructures qui " constituent pour les territoires concernés un désastre écologique, socio-économique et humain ", s'est déroulé en juillet 2013 à Stuttgart après le Val de Suze et Notre-Damedes-Landes.

Il serait utile d'enquêter hors d'Europe pour savoir si la grande vitesse jouit encore des mêmes vertus supposées et rencontre les faveurs de ce type de grand équipement. Ce numéro lève un peu le voile avec le Brésil. Francisco Cortezzi évoque la volonté du gouvernement brésilien de rejoindre la grande vitesse ferroviaire à travers le projet de construction de la première Ligne à Grande Vitesse du pays. L'enjeu est de parcourir $511 \mathrm{~km}$ en 90 minutes, en connectant trois importants pôles urbains et économiques de la région Sud-Est du pays : Rio de Janeiro, São Paulo et Campinas. Ce projet s'inscrit dans un contexte spécifique marqué à la fois par un processus d'urbanisation intense et par une prédominance du transport aérien, automobile et de bus. L'objectif principal du projet est d'améliorer la fluidité des déplacements dans ce corridor mais aussi de participer au développement social et économique dans l'ensemble du pays. Il s'agit aussi de combler les retards et les manques d'investissements dans le transport ferroviaire brésilien. Les grandes attentes reposent sur les mêmes que celles qui ont présidé au développement de la grande vitesse en Europe. Mais s'y ajoute l'ambition originale de revitaliser les gares qui ont été abandonnées et de mailler plus finement les dessertes, sachant qu'il n'existe plus de train de passagers entre les trois villes. Pourtant, ce projet du gouvernement, apparu en 2007, semble piétiner, du fait, principalement, de la difficulté à convaincre le secteur privé. La cause ne viendrait-elle pas du décalage entre les effets attendus du mythe de la technologie à grande vitesse et les besoins territoriaux et sociaux qui pourraient être satisfaits d'une manière plus réaliste et plus utile par le développement des trains régionaux « traditionnels »? Le cas brésilien témoignerait-il lui aussi de l'essoufflement du mythe TGV comme élément de dynamisation des territoires ? La question mériterait un examen approfondi à travers cette non-réalisation qui recèle une vertu heuristique.

Les contributions de ce numéro spécial de la Revue Géographique de l'Est ne sont qu'une pierre d'attente sur le chemin de la construction d'une théorie générale sur l'histoire, les effets et la perception sociale de la grande vitesse ferroviaire sur les territoires européens. Une fois encore est démontré le décalage qui existe entre le discours politique, qui souvent relève de la pensée magique, et l'approche scientifique attachée à mesurer les écarts ou les contradictions qui se produisent inévitablement entre les attentes, les réalisations et les modes d'appropriation et de représentation. Ce qui a été observé sur la question de l'impact ou de l'attractivité, chantier bien avancé, reste à 
accomplir sur la problématique de la gouvernance territoriale et de la dynamique européenne. C'est le moment, à l'heure où la grande vitesse ferroviaire ne semble plus être la voie royale de la modernité européenne.

\section{BIBLIOGRAPHY}

Belot R., Lamard P. (dir.), 2009, Alstom à Belfort. 130 ans d'aventure industrielle, Paris, éd. ETAI.

Belot R., Lamard P., 2013, « Aux origines de la LGV Rhin-Rhône : le rôle de la Suisse dans les logiques argumentatives des stratégies ferroviaires dans le Nord-Franche-Comté (XIX ${ }^{\mathrm{e}}-\mathrm{XXI}^{\mathrm{e}} \mathrm{s}$.) », revue Recherche, Transport et Sécurité, p. 211-227.

Fressoz M., 2011, FGV. Faillite à grande vitesse. 30 ans de TGV, Paris, Le Cherche Midi.

Kosciusko-Moriset N., 2012, Ville Rail et Transports, 25/01/2012.

Troin J.-F., 1997, «Les gares TGV et le territoire : débats et enjeux », Annales de Géographique, $\mathrm{n}^{\circ}$ 593-594, p. 34-50.

\section{AUTHOR}

\section{ROBERT BELOT}

Laboratoire IRTES-RECITS (Institut de recherche sur les transports, l'énergie et la société), EA n ${ }^{\circ}$ 7274, Université de Technologie de Belfort-Montbéliard - robert.belot@utbm.fr 